\title{
Editorial
}

\author{
Melvyn A. Goodale • Francesco Lacquaniti • \\ Carlo A. Marzi
}

Published online: 25 December 2009

(C) Springer-Verlag 2009

January 2010 marks the 200th volume of Experimental Brain Research. When the first slim issue appeared in March 1966, few would have guessed that neuroscience, particularly behavioural and cognitive neuroscience, would see such spectacular growth over the next 40 years. It has been an exciting four decades, and EBR has taken a leading role in charting the way forward, providing an eclectic forum for research across a broad spectrum of neuroscience. EBR has been especially successful at promoting research in brain and behaviour in humans, particularly in neural substrates of sensorimotor control and perception. The articles in this issue highlight some of the innovative new work in this area.

Alan Cowey provides a fascinating as well as thorough critical analysis of the phenomenon of blindsight, that is, the presence of unconscious visual behaviour in the absence of perceptual awareness. Blindsight is examined from many points of view and one of the merits of the review is that it goes deeply into the basic anatomy and

\footnotetext{
M. A. Goodale ( $\square)$

Department of Psychology, University of Western Ontario, London, ON N6A 5C2, Canada

e-mail:mgoodale@uwo.ca

F. Lacquaniti

Department of Neuroscience,

University of Rome “Tor Vergata”, 00173 Rome, Italy

F. Lacquaniti

Human Physiology Section, Scientific Institute Santa Lucia, Via Ardeatina 306, 00179 Rome, Italy

\section{A. Marzi}

Department of Neurological Science and Vision Physiology Section, University of Verona, 8 Strada Le Grazie,

37134 Verona, Italy
}

neurology of the loss of V1, often making reference to the extensive pioneering work of the author on non-human primates. Cowey's attitude to blindsight (which he dubs a "saga" in the title) is twofold: on one hand he is constructively critical; on the other, he is clearly fascinated by its philosophical as well as practical implications. Among the specific merits of this paper, we would like to highlight the diagram in Figure 2 comparing the visual pathways in the normal brain to those remaining after a lesion of V1. The diagram provides an intriguing roadmap for planning new blindsight work-and by the same token, the list of things still to do in blindsight research gives concrete examples of where research might go next.

Giovanni Berlucchi and Salvatore Aglioti review the exciting work on the neural representation of the body, tackling some of the controversies surrounding the concepts of body image and body schema. As they point out, not only does our brain enable us to experience our body (and our sense of self) but it also uses these mechanisms in constructing our perception and understanding of others. Berlucchi and Aglioti, who have done some of the ground-breaking work in this area themselves, examine the validity of the confusing but oft-made distinction between body image and body schema - and evaluate some of the more recent dissociations and distinctions that have been put forward. They provide a succinct and useful review of the plethora of studies that have explored the role of various brain areas like the extrastriate and fusiform body areas as well as regions within the parietal cortex and insula. But as they emphasize at the end of their review, the existence of these different cortical areas and their associated somatotopies suggests that there are many representations of the body in the brain, but does not explain how the integrated and continuous sense that we have of our body and our self is generated. As they conclude, there is plenty of work to be done. 
The article by Vidal and Bülthoff fills an important gap in the vast literature on multi-sensory integration for the perception of self-motion. Using a sophisticated display system, they manipulated vestibular and visual cues independently. The results of their experiment were crystal clear. They showed that vestibular and visual information about whole-body turning movements are stored independently, but they can be combined in recalling that turn. These and other findings offer new insights into how vision and other sensory systems allow us to compute changes in body position and heading. The neural coding of heading was also the issue that motivated the electrophysiological study by Bremmer, Kubischik, Pekel, Hoffman, and Lappe. These investigators recorded the activity of neurons in the medio-superior temporal area (MST) in the monkey in response to different optic flow fields. They showed that some MST neurons were not only sensitive to radial flow fields, simulating a particular heading, but these cells also compensated for distortions in the field that might arise if the animal moved its eyes. In different ways, both these studies provide important clues into the way in which our brain codes and controls our movements over time.

The article by Anderson, Mannan, Rees, Sumner, and Kennard combines a behavioural dual-task paradigm with functional magnetic resonance imaging (fMRI). These authors tracked the cortical regions common to inefficient visual search and to both spatial and non-spatial working memory. Their conclusion is that overlapping regions in inferior and middle frontal cortex of the right hemisphere are important for both types of task. Thus, attentional selection from memory and handling information in memory converge in these same areas.

In a simple but elegant experiment, Pia, Neppi-Modona, and Folegatti show that pseudoneglect, the slight leftward error in the bisection of a horizontal line that healthy subjects typically commit, is object- rather than viewer-centred. They used stimuli (i.e. an elongated caricature of a basset hound) similar to that employed by Savazzi et al. (Brain Res Cogn Brain Res 18:234-241, 2004) with neglect patients. Pia et al. found that pseudoneglect reversed from left to right in the critical trials in which the image was mirror reversed thus showing that it is possible to induce an object-centred pseudoneglect, which is typically found with words, with non-verbal stimuli as well. This result is conceptually akin to that obtained by Savazzi et al. (2004) with neglect patients and this suggests that the neural mechanism underlying neglect and pseudoneglect are similar.

Prodoehl and Vaillancourt provide new evidence that changes in visual gain are important for the control of isometric force output at the elbow and ankle, that is, at large joints-while previous studies have focused on the fingers. Moreover, Prodoehl and Vaillancourt show that changes in visual gain increased variability and structure of force out- put more at the elbow than at the ankle. Interestingly, this improved control was related to changes in the pattern of agonist and antagonist muscle activation.

The article by Galie and Jones describes experiments that explored the intriguing possibility that the thermal cues generated on the fingertips as an object is grasped could influence the perception of force and the perceived weight of the object. When we pick up an object, heat is transferred by conduction from our fingertips to the object, which is typically cooler than our hand. Galie and Jones first demonstrated a systematic relationship between applied force and the change in temperature at the fingertips. They then went onto show that changing the temperature of the object to which the forces were being applied had no effect on the perceived magnitude of the fingertip forces. Importantly, their results suggest that thermal cues play very different roles in the perception of actively generated forces than they do in more passive situations, where the weights of objects are perceived tactually, offering some support for a centrally generated theory of force perception.

Although there have been a number of demonstrations of predictive re-mapping of receptive fields using single-cell recording in the monkey, Mathot and Theeuwes investigated this phenomenon for the first time in humans by looking at how attention changes prior to a saccadic eye movement. In their cleverly designed experiments, they showed that the re-mapping that is presumed to occur just before the execution of saccade results in attentional facilitation at a location that would end up being the attended location after the eye movement. As they put it, this remapping delivers a kind of "status report" about a location in the world that will be brought into the receptive field of a neuron by the eye movement. Such re-mapping clearly has consequences for the allocation of attention.

Finally, the article by Rossit, Szymanek, Butler, and Harvey addresses the issue of whether or not memoryguided saccades, unlike saccades made to visible targets, depend on mechanisms within the ventral 'perception' stream. They tackled this question by looking at memoryguided saccades in patient DF, who is without doubt the most highly studied patient with ventral-stream damage in the world. Although DF, who has large bilateral lesions in the lateral occipital cortex, was able to direct her saccades to visible targets as well as neurologically intact individuals could, her memory-guided saccades were much less accurate. These results lend further support to the idea that the off-line control of actions cannot be mediated by the dorsal 'action' pathway by itself but requires the participation of perceptual mechanisms within the ventral stream.

We believe that this issue of EBR's 200th volume not only provides a cross section of some of the best work going on today in behavioural and cognitive neuroscience, but (hopefully) sets the stage for the next 40 years of research. 